\title{
Prophylactic pyridoxine was not able to reduce the incidence of capecitabine-induced hand-foot syndrome: A meta-analysis
}

\author{
YUN ZHOU $^{1}$, LING PENG $^{2}$, YINGJIE LI ${ }^{1}$ and LIXUN CHEN ${ }^{1}$ \\ ${ }^{1}$ Zhejiang Food and Drug Administration; ${ }^{2}$ Department of Thoracic Oncology, The First Affiliated Hospital, \\ School of Medicine, Zhejiang University, Hangzhou, Zhejiang 310003, P.R. China
}

Received May 10, 2013; Accepted July 02, 2013

DOI: $10.3892 /$ br.2013.161

\begin{abstract}
Hand-foot syndrome (HFS) is a dose-limiting adverse event of capecitabine, which commonly leads to early discontinuation of capecitabine-based therapy in the palliative and adjuvant settings. Although pyridoxine has been used for the prevention of capecitabine-associated HFS, its efficacy is controversial. The aim of this study was to evaluate whether prophylactic pyridoxine reduces the incidence of capecitabine-associated HFS by performing a meta-analysis of the literature involving available studies. Systematic searches for trials were undertaken through PubMed, Embase, Web of Science, the Cochrane Library, the American Society of Clinical Oncology (ASCO) and the ASCO Gastrointestinal Cancers Symposium, updated to March, 2013, to identify relevant studies. A meta-analysis was conducted with eligible studies that evaluated the efficacy of the prophylactic use of pyridoxine against capecitabine-induced HFS. We performed a meta-analysis of five studies ( $n=793$ patients) that evaluated the efficacy of the prophylactic use of pyridoxine in cancer patients treated with capecitabine. The odds ratio (OR) comparing prophylactic pyridoxine to placebo was 0.91 [95\% confidence interval (CI): 0.67-1.24] for HFS of all grades; OR=1.17 (95\% CI: 0.82-1.67) for $\mathrm{HFS} \geq$ grade 2 and $\mathrm{OR}=1.05$ (95\% CI: $0.60-1.85$ ) for HFS $\geq$ grade 3 . Based on our meta-analysis, prophylactic pyridoxine did not appear to reduce the incidence of HFS in patients receiving capecitabine.
\end{abstract}

\section{Introduction}

Capecitabine $\left(\mathrm{Xeloda}^{\circledR}\right)$, an oral fluoropyrimidine, is a systemic prodrug of 5-fluorouracil (5-FU), with the advantage of oral vs. intravenous administration, which is required for 5-FU.

Correspondence to: Dr Ling Peng, Department of Thoracic Oncology, The First Affiliated Hospital, School of Medicine, Zhejiang University, 79 Qingchun Road, Hangzhou, Zhejiang 310003, P.R. China

E-mail: dr.pengling@gmail.com

Key words: capecitabine, hand-foot syndrome, pyridoxine, prophylactic, meta-analysis
Capecitabine is effective in the treatment of various malignancies, including colorectal, breast and gastric cancer (1-4). Although capecitabine is generally well-tolerated, hand-foot syndrome (HFS) is the most common adverse event associated with capecitabine and was proven to be a chronic dose-limiting toxicity, leading to significant morbidity in patients receiving this type of treatment (5).

HFS, also referred to as palmar-plantar erythrodysesthesia (PPE), is a distinctive toxic reaction associated with certain chemotherapeutic agents (6). The incidence of HFS associated with capecitabine is $50-60 \%$ and the incidence of severe HFS ( $\geq$ grade 3 ) may be $10-70 \%$ (7). HFS is dose-dependent and its occurrence is determined by peak drug concentration and total cumulative dose (8). The mechanism underlying the development of HFS has not been elucidated, although the majority of researchers consider it to be an inflammatory reaction. The mainstay of the management of HFS is temporary treatment interruption and, if necessary, dose reduction. Topical agents, such as potent topical steroids and emollients are occasionally used to reduce pain and discomfort and protect against infections.

Since HFS was found to resemble a rat disease (acrodynia) caused by pyridoxine (vitamin B6) deficiency, capecitabine-associated HFS was empirically treated with pyridoxine $(9,10)$. Several studies investigated whether pyridoxine is able to reduce the incidence of capecitabine-induced HFS (15,16,30-32). However, the results of those studies were inconclusive and no consensus was reached. The purpose of our study was to determine whether pyridoxine therapy is able to prevent the development of HFS in patients being treated with capecitabine. In addition, we performed analyses of publication bias and heterogeneity between published studies.

\section{Materials and methods}

Search strategy and study selection. The following databases were searched using the medical subject headings and search tags 'capecitabine' or 'Xeloda', 'pyridoxine' or 'vitamin B6', 'hand-foot syndrome' or 'palmar-plantar erythrodysesthesia': PubMed, Embase, Web of Science, the Cochrane Library, the American Society of Clinical Oncology (ASCO) and the ASCO Gastrointestinal Cancers Symposium. The upper date limit of March, 2013 was applied, with no lower date limit. A manual search for general reviews on HFS associated with 


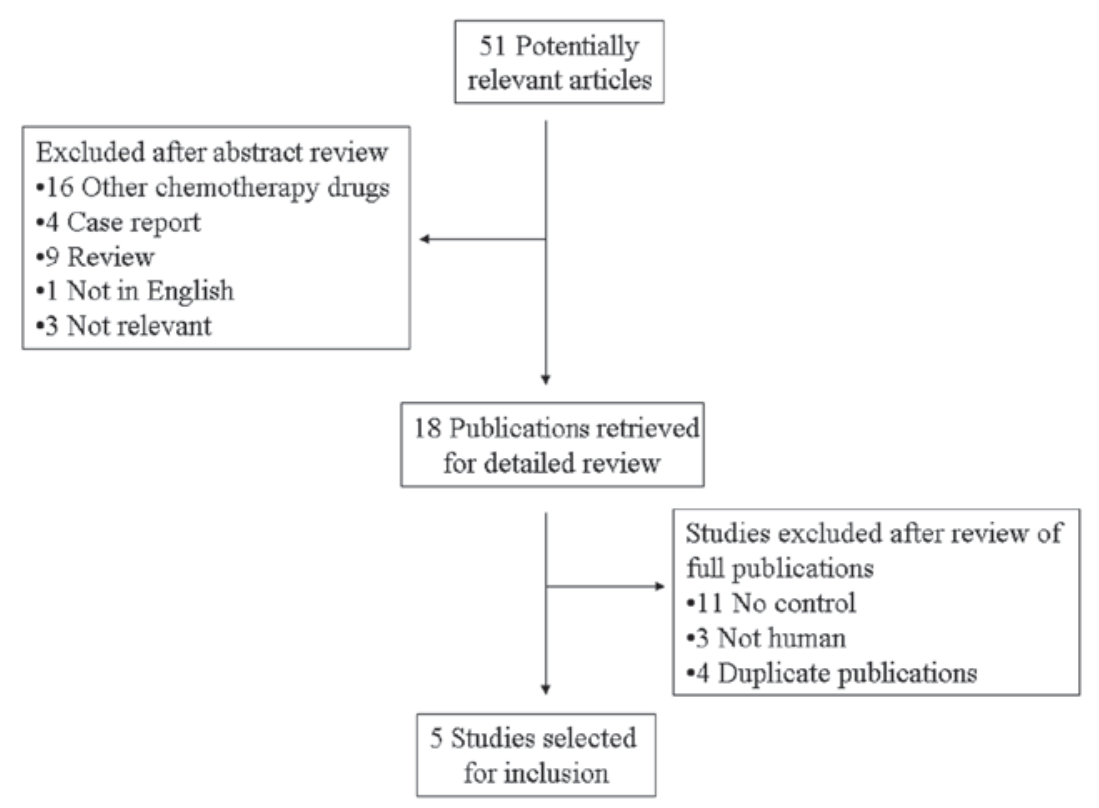

Figure 1. Flow chart of search strategy.

capecitabine and references cited by the included studies were also used to complete the search. The search results were downloaded to reference databases and then screened.

In order to be eligible for inclusion in this meta-analysis, a study was required to meet the following criteria: i) the patients received chemotherapy that included capecitabine; ii) the trials included a treatment group receiving pyridoxine during chemotherapy and a control group that did not receive pyridoxine; iii) the evaluation of HFS in the published trials adopted the toxicity grading of the National Cancer Institute Common Toxicity Criteria (NCI-CTC). Trials were excluded if they did not meet the criteria described above. Additional exclusion criteria included the following: i) animal or in vitro studies; ii) not primary studies (e.g., review articles, letters to the editor); or iii) duplicate publications of other studies previously identified in our systematic evaluation. To identify multiple publications from the same data sets, we investigated all author names, different institutions involved and the time period of patient recruitment of each study. When the same author reported results from the same patient population, the most recent or the most complete study was included. The abstracts of all candidate studies were read by two independent readers (L.P. and Y.Z.). Studies that could not be classified based on the title and abstract alone were retrieved for full-text review. Disagreements were resolved by consensus between the two readers.

Data extraction. The final studies included were independently assessed by two readers (L.P. and Y.Z.). Information was carefully retrieved from the studies, using a standardized data collection form, including the following items: first author, year of publication, country of origin, number of patients allocated, patient characteristics and chemotherapeutic regimen. If data from any of the above categories were not reported in the study, the items were treated as 'not specified'. To ensure accuracy, targeted data were extracted by two authors working independently. If the study had more than one grade criteria, only data regarding HFS incidence graded on the basis of the NCI-CTC were extracted. The authors of the primary studies were not contacted for additional or unreported information. We did not use prespecified quality-related inclusion or exclusion criteria and did not weigh each study by a quality score, since the quality score has not received general approval for use in meta-analyses, particularly observational studies.

Statistical methods. The primary outcome for analysis was the incidence of HFS in patients receiving capecitabine. Dichotomous variables were analyzed with odds ratios (ORs) with mean differences at a $95 \%$ confidence interval (CI). The calculations were performed under the hypothesis that the variance of the two groups was identical (11). Two-sided P-values were computed for the differences between dichotomous variables, which were considered significant at $\mathrm{P}<0.05$. The heterogeneity of the individual ORs was calculated with $\chi^{2}$ tests according to Peto's method (12). A heterogeneity test with inconsistency index $\left(\mathrm{I}^{2}\right)$ statistic and $\mathrm{Q}$ statistic was performed. If $\mathrm{P}>0.10$ or $\mathrm{I}^{2} \leq 50 \%$, the heterogeneity of the trial was considered acceptable and the differences between the OR and $95 \%$ CI were computed by the fixed-effects model. If $\mathrm{P} \leq 0.10$ or $\mathrm{I}^{2}>50 \%$, the differences between the OR and $95 \% \mathrm{CI}$ were computed by the random-effects model.

Publication bias was identified with funnel plots $(13,14)$, whereby asymmetries in the funnel plot indicated publication bias. Intercept significance was determined by the t-test suggested by Egger $(\mathrm{P}<0.05$ was considered representative of statistically significant publication bias). All the calculations were performed by Stata software version 11.0 (StataCorp, College Station, TX, USA).

\section{Results}

Study selection and characteristics. A total of 51 potentially relevant citations were reviewed and five studies met the 
Table I. Main characteristics and results of the eligible studies.

\begin{tabular}{|c|c|c|c|c|c|c|c|c|}
\hline \multirow[b]{2}{*}{ Study } & \multirow[b]{2}{*}{ Country } & \multirow[b]{2}{*}{ Site of tumor } & \multirow[b]{2}{*}{ Type of study } & \multirow{2}{*}{$\begin{array}{l}\text { Chemotherapy } \\
\text { regimen }\end{array}$} & \multicolumn{2}{|c|}{ Enrolled patients } & \multirow{2}{*}{$\begin{array}{c}\text { Dose of } \\
\text { pyridoxine } \\
(\mathrm{mg} / \text { day })\end{array}$} & \multirow[b]{2}{*}{ Refs } \\
\hline & & & & & Pyridoxine & Control & & \\
\hline Mortimer et al & United States & Colon, breast & Retrospective & NS & 73 & 99 & $50-600$ & (15) \\
\hline Yoshimoto et al & Japan & NS & Retrospective & $\begin{array}{l}\text { X or X with } \\
\text { CTX and EPI }\end{array}$ & 38 & 40 & 60 & (30) \\
\hline Kang et al & Korea & $\begin{array}{l}\text { Stomach, colon, } \\
\text { biliary tract, duodenum }\end{array}$ & $\mathrm{RCT}$ & X, XP, DXP & 180 & 180 & 200 & (31) \\
\hline Braik et al & United States & NS & $\mathrm{RCT}$ & NS & 38 & 39 & 100 & (32) \\
\hline Corrie et al & United Kingdom & Colorectum, breast & $\mathrm{RCT}$ & NS & 53 & 53 & 150 & (16) \\
\hline
\end{tabular}

Summary table of studies included in the meta-analysis. NS, not specified; X, Xeloda ${ }^{\circledR}$ (capecitabine); CTX, cyclophosphamide; EPI, epirubicin; RCT, randomized controlled trial; XP, capecitabine and cisplatin; DXP, docetaxel, capecitabine and cisplatin.

Table II. HFS grading according to the National Cancer Institute Common Toxicity Criteria (NCI-CTC) version 4.0.

Severity Criteria

Grade $1 \quad$ Minimal skin changes or dermatitis (e.g., erythema, edema or hyperkeratosis) without pain

Grade 2 Skin changes (e.g., peeling, blisters, bleeding, edema or hyperkeratosis) with pain; limiting instrumental ADL

Grade 3 Severe skin changes (e.g., peeling, blisters, bleeding, edema or hyperkeratosis) with pain; limiting self care ADL

HFS, hand-foot syndrome; ADL, activities of daily living.

inclusion criteria in the search strategy and study selection section, comprising 793 patients for the final analysis (Fig. 1). Of the five studies, three were prospective randomized control trials, whereas the remaining two were retrospective studies. The major baseline characteristics of the five eligible publications are presented in Table I. The studies were conducted in four countries (Japan, Korea, the United States of America and the United Kingdom) and were published between 2003 and 2012. The dose of pyridoxine varied from 50 to $600 \mathrm{mg} / \mathrm{day}$. All the studies reported the incidence of HFS in patients receiving capecitabine. As regards chemotherapy outcome, two trials $(15,16)$ compared the treatment outcome between the two groups according to the response evaluation criteria in solid tumors (17). The characteristics of the included trials are listed in Table I. The NCI-CTC criteria are listed in Table II.

Meta-analysis. The results of the meta-analysis are shown in Fig. 2. The five trials included in this study evaluated capecitabine-induced HFS according to the NCI-CTC. The pooled analysis revealed that, compared to the placebo, pyridoxine did not reduce the number of patients with HFS of all grades, with an OR of 0.91 (95\% CI: 0.67-1.24). The combined ORs for the studies evaluating the prophylactic use of pyridoxine in preventing capecitabine-associated HFS of $\geq$ grade 2 and severe ( $\geq$ grade 3 ) were 1.17 (95\% CI: 0.82-1.67) and 1.05 (95\% CI: $0.60-1.85$ ), respectively, indicating that pyridoxine was not effective in the prevention against HFS of any grade. No significant heterogeneity was observed among the studies (all grades: $\mathrm{Q}=9.55, \mathrm{I}^{2}=58.1 \%, \mathrm{P}=0.049 ; \geq$ grade 2: $\mathrm{Q}=1.74, \mathrm{I}^{2}=0.0 \%, \mathrm{P}=0.628$; and $\geq$ grade $3: \mathrm{Q}=3.44, \mathrm{I}^{2}=0.0 \%$, $\mathrm{P}=0.487)$.

Treatment outcomes of chemotherapy. The effects of chemotherapy on unresectable tumors in the placebo and pyridoxine groups were compared between two trials. No significant differences were observed in the response rates, disease control rates or survival times between the two groups. The results indicated that the use of pyridoxine did not appear to increase the recurrence of tumors.

Publication bias. Begg's funnel plot and Egger's test were performed to evaluate the publication bias of the eligible studies (Fig. 3). The Egger's test score of pyridoxine in the prevention against capecitabine-induced HFS of all grades, $\geq$ grade 2 and severe ( $\geq$ grade 3 ) yielded P-values of $0.200,0.11$ and 0.016 , respectively, indicating that there was publication bias in severe HFS.

\section{Discussion}

Capecitabine is an oral fluoropyrimidine that is used to treat various types of cancer. Chemotherapy regimens including capecitabine are currently considered to be a standard of care in the management of colorectal, breast and gastric cancers. However, capecitabine-induced HFS is a commonly occurring adverse event that affects the quality of life of the patients and may lead to postponement or even interruption 


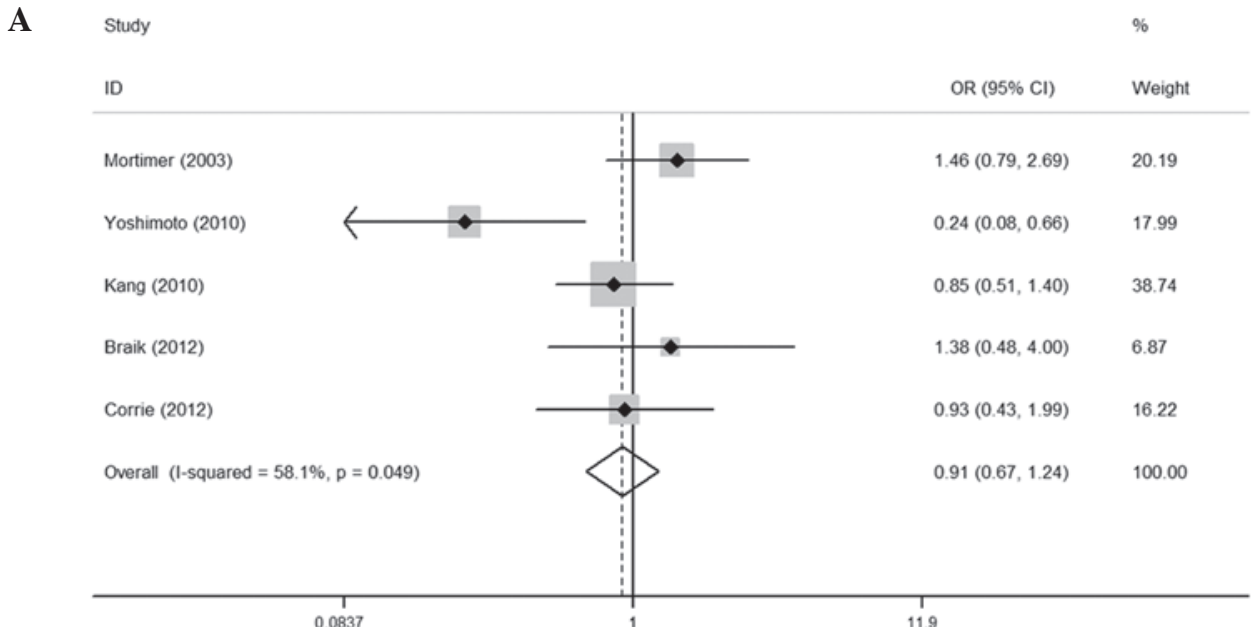

B
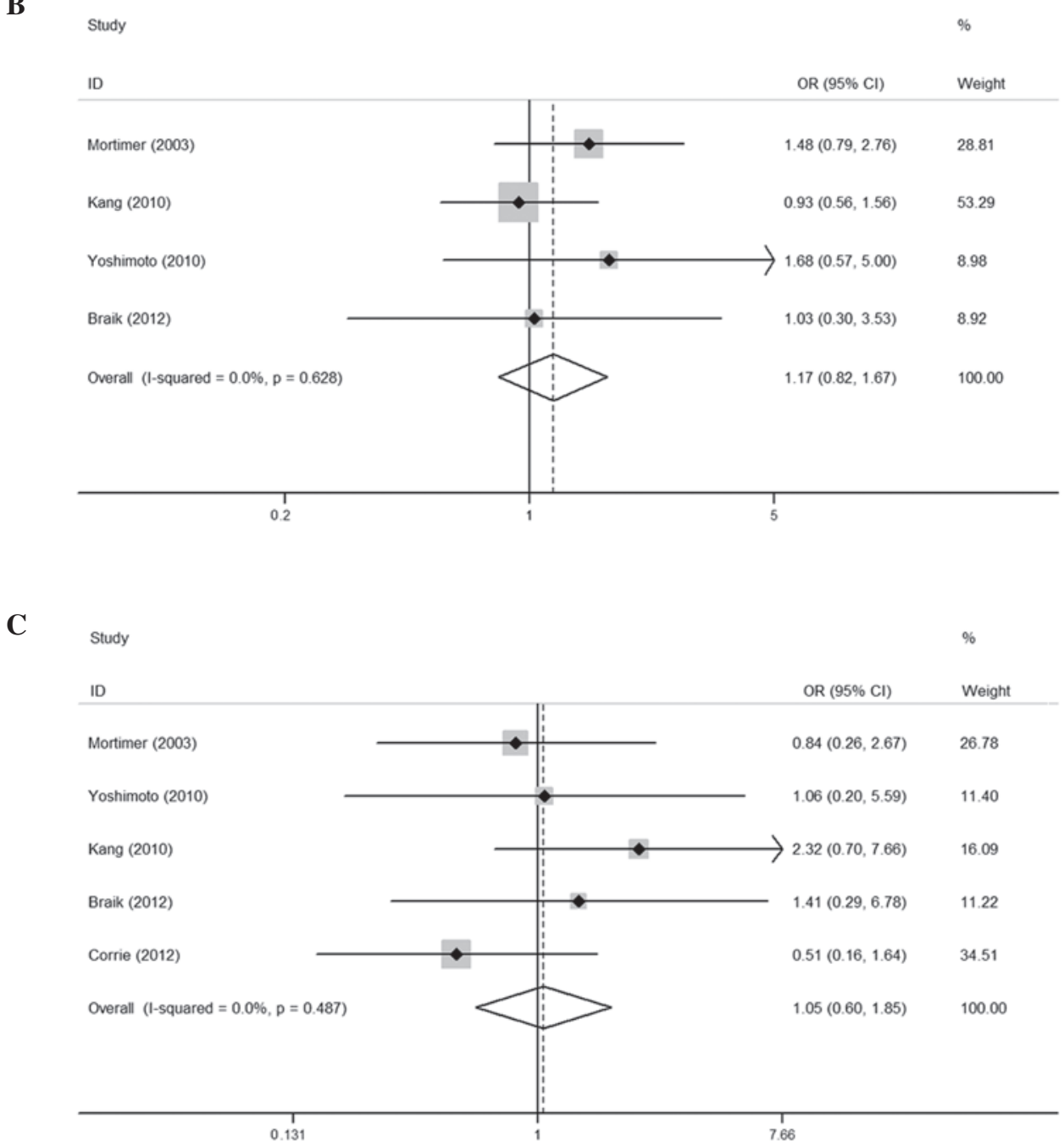

Figure 2. Meta-analysis (forest plot) of the eligible studies.

of capecitabine therapy. Hand-foot syndrome, also referred to as palmar-plantar erythrodysesthesia or palmoplantar keratoderma, is a dose-limiting adverse effect associated with capecitabine. Several chemotherapeutic drugs have been identified as a cause of HFS, such as doxorubicin, liposomal doxorubicin, docetaxel and fluoropyrimidine (18-21). The clinical manifestations present as dysesthesia, followed by painful, symmetric, well-defined erythema and edema. The median time of onset is 79 days, although the range may be 11-360 days (22). HFS is an emerging issue in capecitabine cancer treatment, leading to additional morbidity, suboptimal dosing and poor compliance with treatment. 

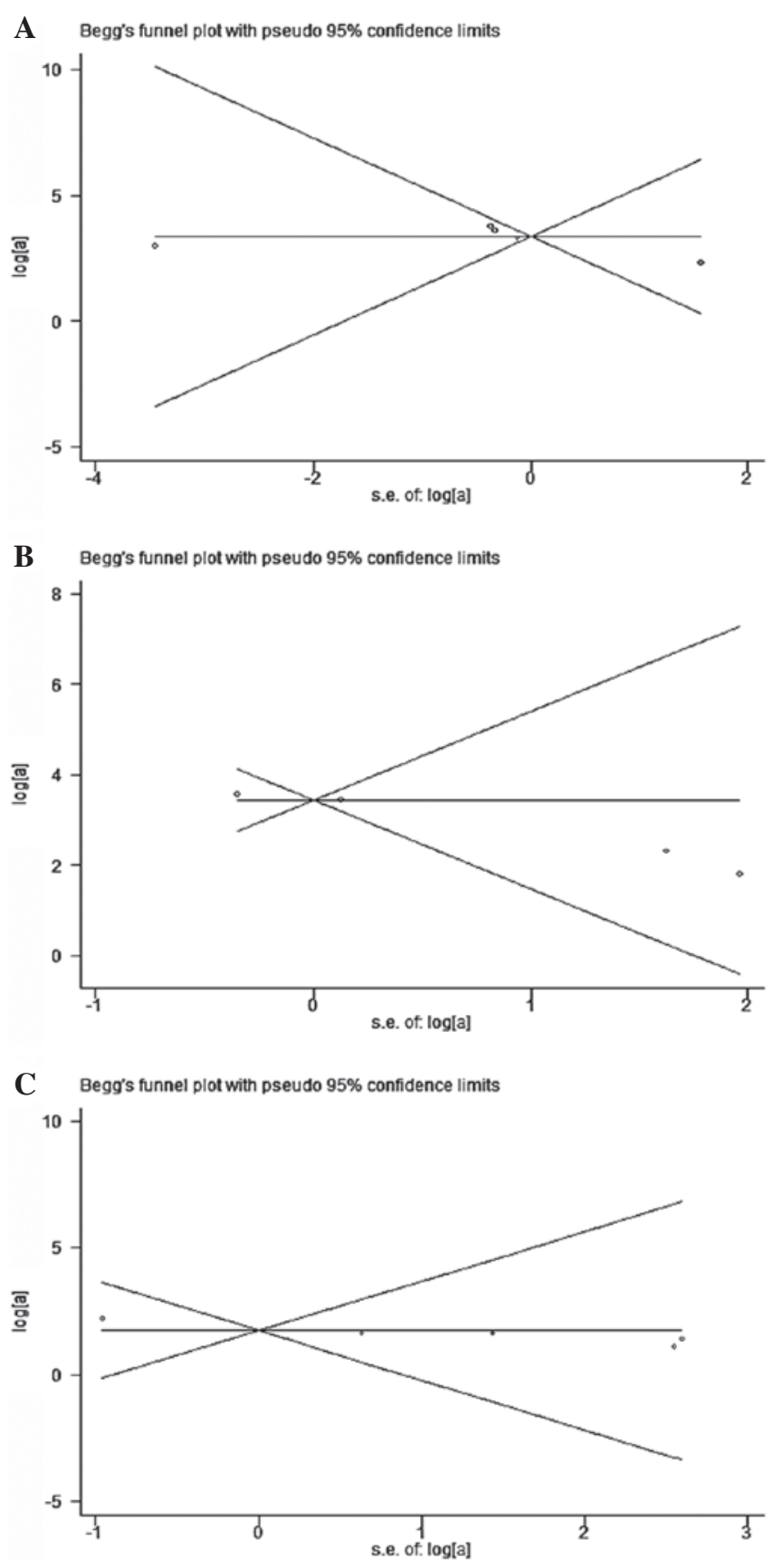

Figure 3. Funnel plot for studies included in the meta-analysis.

The mechanism underlying the development of HFS has not been elucidated. One suggested hypothesis was that the keratinocytes in the skin may upregulate the enzyme thymidine phosphorylase, which increases the accumulation of capecitabine metabolites. Another hypothesis was that capecitabine may be excreted by sweat glands, which are profuse in the palms and the soles (7). It was also hypothesized that HFS is induced by capecitabine metabolites through a prostaglandin-like action (23).

The treatment for capecitabine-induced HFS is dose reduction or permanent discontinuation of capecitabine. Topical agents, such as steroids, emollients and 99\% dimethyl sulfoxide are occasionally used for relief (24). Since HFS was found to resemble a rat disease (acrodynia) caused by pyridoxine deficiency, capecitabine-induced HFS was empirically treated with pyridoxine. Animal studies, case reports and other small studies previously suggested that the administration of pyridoxine may help prevent and treat HFS induced by chemotherapy drugs $(9,10,25-27)$. It appears to be effective in preventing against HFS in at least some individuals who experience this side effect. As pyridoxine is a safe nutritional supplement, its prophylactic use seems appealing. Pyridoxine is often used to treat HFS associated with capecitabine; however, the evidence regarding its beneficial effect is not adequate. There is no consensus to prove that pyridoxine prevents or treats HFS. Therefore, the present meta-analysis was conducted to determine the effect of prophylactic pyridoxine administration in reducing the incidence of HFS in patients receiving capecitabine.

The present meta-analysis, which was based on data provided by five studies including a total of 793 patients to yield statistics, compared the administration of pyridoxine to placebo regarding the prevention of capecitabine-induced HFS. Pyridoxine did not provide any marked benefit against capecitabine-induced HFS. An important issue in these studies was that the dose of pyridoxine varied from 50 to $600 \mathrm{mg} /$ day. Long-term use of high doses of pyridoxine may lead to the development of additional side effects or health problems or interfere with the absorption and use of other important nutrients. Since a preclinical model or pharmacodynamic data on the dose-response relationship of pyridoxine are not available, the optimal dose of pyridoxine cannot be determined.

Another controversial issue was the possible effect of pyridoxine on tumor response and patient survival. The investigation on the correlation between HFS and clinical outcome has been limited. It was suggested that patients who were administered high doses of pyridoxine exhibited worse tumor response (26). In our meta-analysis, two of the primary studies evaluated tumor response in the pyridoxine vs. placebo groups, which revealed no difference in the tumor response rate. This may be explained by the different doses of pyridoxine used in the studies, which requires further investigation.

Our meta-analysis has several limitations. First, two of the included studies were not randomized controlled trials but retrospective studies. Retrospective studies limit confidence in the further clinical utility of pyridoxine. Second, although there was no significant heterogeneity among the primary studies, it should be noted that, due to the small number of primary studies analyzed in each group, the power of detection of potentially important differences was limited. Third, the meta-analysis relied on publication, rather than individual patient data (IPD). Therefore, the results must be interpreted with caution, since the IPD-based analysis provides the least bias and is more reliable compared to the literature-based meta-analysis (28).

Biases should also be handled with caution. First, publication bias is a major concern in all forms of meta-analysis, as published studies are often positive (29). As for the results of insignificant publication bias, it should be noted that when the sample size of the studies or the number of eligible studies is limited, the power of detecting publication bias by the linear regression model is reduced. Considering the limited number of studies included, the findings from our meta-analysis require confirmation by further studies. Furthermore, the language bias could not be completely avoided, as the inclusion criteria was restricted to studies published in English. A 
selection process with rigid inclusion criteria was adopted in ascertaining studies, thereby reducing selection bias.

In conclusion, to the best of our knowledge, our meta-analysis is the first study to systematically evaluate the efficacy of prophylactic pyridoxine in preventing capecitabine-induced HFS. Our results led to the conclusion that pyridoxine was not able to prevent capecitabine-induced HFS. Therefore, the use of pyridoxine may not be currently recommended for prophylaxis against HFS. To strengthen our findings, well-designed prospective studies may help determine the efficacy of pyridoxine in the prevention of capecitabine-induced HFS.

\section{Acknowledgements}

We are indebted to the authors of the primary studies. This study was supported by the Medical Science Research Foundation of the Health Bureau of Zhejiang Province (no. 2012KYA072) and the Administration of Traditional Chinese Medicine of Zhejiang Province (no. 2012ZB084).

\section{References}

1. Van Cutsem E, Twelves C, Cassidy J, et al: Oral capecitabine compared with intravenous fluorouracil plus leucovorin in patients with metastatic colorectal cancer: results of a large phase III study. J Clin Oncol 19: 4097-4106, 2001.

2. Blum JL, Dieras V, Lo Russo PM, et al: Multicenter, phase II study of capecitabine in taxane-pretreated metastatic breast carcinoma patients. Cancer 92: 1759-1768, 2001.

3. Twelves C, Wong A, Nowacki MP, et al: Capecitabine as adjuvant treatment for stage III colon cancer. N Engl J Med 352: 2696-2704, 2005.

4. Bang YJ: Capecitabine in gastric cancer. Expert Rev Anticancer Ther 11: 1791-1806, 2011

5. Scheithauer W and Blum J: Coming to grips with hand-foot syndrome. Insights from clinical trials evaluating capecitabine. Oncology (Williston Park) 18: 1161-1168; discussion 1173-1176, $1181-1184,2004$

6. Nagore E, Insa A and Sanmartin O: Antineoplastic therapy-induced palmar plantar erythrodysesthesia ('hand-foot') syndrome. Incidence, recognition and management. Am J Clin Dermatol 1: 225-234, 2000.

7. Gressett SM, Stanford BL and Hardwicke F: Management of hand-foot syndrome induced by capecitabine. J Oncol Pharm Pract 12: 131-141, 2006.

8. Chiara S, Nobile MT, Barzacchi C, et al: Hand-foot syndrome induced by high-dose, short-term, continuous 5-fluorouracil infusion. Eur J Cancer 33: 967-969, 1997.

9. Vukelja SJ, Lombardo FA, James WD and Weiss RB: Pyridoxine for the palmar-plantar erythrodysesthesia syndrome. Ann Intern Med 111: 688-689, 1989.

10. Fabian CJ, Molina R, Slavik M, Dahlberg S, Giri S and Stephens R: Pyridoxine therapy for palmar-plantar erythrodysesthesia associated with continuous 5-fluorouracil infusion. Invest New Drugs 8: 57-63, 1990.

11. DerSimonian R and Laird N: Meta-analysis in clinical trials. Control Clin Trials 7: 177-188, 1986.

12. Yusuf S, Peto R, Lewis J, Collins R and Sleight P: Beta blockade during and after myocardial infarction: an overview of the randomized trials. Prog Cardiovasc Dis 27: 335-371, 1985.

13. Egger M, Davey Smith G, Schneider M and Minder C: Bias in meta-analysis detected by a simple, graphical test. BMJ 315 629-634, 1997.
14. Macaskill P, Walter SD and Irwig L: A comparison of methods to detect publication bias in meta-analysis. Stat Med 20: 641-654, 2001.

15. Mortimer JE, Lauman MK, Tan B, Dempsey CL, Shillington AC and Hutchins KS: Pyridoxine treatment and prevention of hand-and-foot syndrome in patients receiving capecitabine. J Oncol Pharm Pract 9: 161-166, 2003.

16. Corrie PG, Bulusu R, Wilson CB, et al: A randomised study evaluating the use of pyridoxine to avoid capecitabine dose modifications. Br J Cancer 107: 585-587, 2012

17. Therasse P, Arbuck SG, Eisenhauer EA, et al: New guidelines to evaluate the response to treatment in solid tumors. European Organization for Research and Treatment of Cancer, National Cancer Institute of the United States, National Cancer Institute of Canada. J Natl Cancer Inst 92: 205-216, 2000.

18. Lokich JJ and Moore C: Chemotherapy-associated palmar-plantar erythrodysesthesia syndrome. Ann Intern Med 101: 798-799, 1984.

19. Banfield GK, Crate ID and Griffiths CL: Long-term sequelae of palmar-plantar erythrodysaesthesia syndrome secondary to 5-fluorouracil therapy. J R Soc Med 88: 356P-357P, 1995.

20. Zimmerman GC, Keeling JH, Burris HA, et al: Acute cutaneous reactions to docetaxel, a new chemotherapeutic agent. Arch Dermatol 131: 202-206, 1995

21. Gordon KB, Tajuddin A, Guitart J, Kuzel TM, Eramo LR and VonRoenn J: Hand-foot syndrome associated with liposomeencapsulated doxorubicin therapy. Cancer 75: 2169-2173, 1995.

22. Abushullaih S, Saad ED, Munsell M and Hoff PM: Incidence and severity of hand-foot syndrome in colorectal cancer patients treated with capecitabine: a single-institution experience. Cancer Invest 20: 3-10, 2002.

23. Lin E, Morris JS and Ayers GD: Effect of celecoxib on capecitabine-induced hand-foot syndrome and antitumor activity. Oncology (Williston Park) 16: 31-37, 2002.

24. Lopez AM, Wallace L, Dorr RT, Koff M, Hersh EM and Alberts DS: Topical DMSO treatment for pegylated liposomal doxorubicin-induced palmar-plantar erythrodysesthesia. Cancer Chemother Pharmacol 44: 303-306, 1999.

25. Andres R, Mayordomo JI, Lara R, et al: Gemcitabine/capecitabine in patients with metastatic breast cancer pretreated with anthracyclines and taxanes. Clin Breast Cancer 6: 158-162, 2005.

26. Chalermchai T, Tantiphlachiva K, Suwanrusme H, Voravud N and Sriuranpong V: Randomized trial of two different doses of pyridoxine in the prevention of capecitabine-associated palmar-plantar erythrodysesthesia. Asia Pac J Clin Oncol 6: 155-160, 2010.

27. Rossi D and Catalano G: Pyridoxine as prophylactic therapy for palmar-plantar ery throdysesthesia associated with administration of pegylated liposomal doxorubicin (caelyx): a single-center experience. Oncology 73: 277-278, 2007.

28. Stewart LA and Parmar MK: Meta-analysis of the literature or of individual patient data: is there a difference? Lancet 341 : 418-422, 1993.

29. Begg CB and Mazumdar M: Operating characteristics of a rank correlation test for publication bias. Biometrics 50: 1088-1101, 1994.

30. Yoshimoto N, Yamashita T, Fujita T, et al: Impact of prophylactic pyridoxine on occurrence of hand-foot syndrome in patients receiving capecitabine for advanced or metastatic breast cancer. Breast Cancer 17: 298-302, 2010.

31. Kang YK, Lee SS, Yoon DH, et al: Pyridoxine is not effective to prevent hand-foot syndrome associated with capecitabine therapy: results of a randomized, double-blind, placebo-controlled study. J Clin Oncol 28: 3824-3829, 2010.

32. Braik T, Yim B, Evans AT, et al: A randomized trial to determine if vitamin B6 can prevent hand and foot syndrome in cancer patients treated with capecitabine chemotherapy. J Clin Oncol 30 (Suppl; abstr 9085), 2012. 